\title{
SAMPLING OF ENTIRE FUNCTIONS OF SEVERAL COMPLEX VARIABLES ON A LATTICE AND MULTIVARIATE GABOR FRAMES
}

\author{
KARLHEINZ GRÖCHENIG AND YURII LYUBARSKII
}

\begin{abstract}
We give a general construction of entire functions in $d$ complex variables that vanish on a lattice of the form $\Lambda=A(\mathbb{Z}+i \mathbb{Z})^{d}$ for an invertible complex-valued matrix. As an application we exhibit a class of lattices of density $>1$ that fail to be a sampling set for the Bargmann-Fock space in $\mathbb{C}^{2}$. By using an equivalent real-variable formulation, we show that these lattices fail to generate a Gabor frame.
\end{abstract}

\section{INTRODUCTION}

We study the sampling problem in the Bargmann-Fock space of several complex variables and the related construction of Gabor frames with a Gaussian window. Our main point is the restriction to sampling on a lattice in $\mathbb{C}^{d}$ and the consequences resulting from the additional invariance properties.

The first problem is a sampling problem for entire functions in several complex variables. Recall that the Bargmann-Fock space $\mathcal{F}_{d}^{2}$ consists of all entire functions of $d$ complex variables $z=\left(z_{1}, \ldots, z_{d}\right) \in \mathbb{C}^{d}$ with finite norm

$$
\|F\|_{\mathcal{F}_{d}^{2}}^{2}=\int_{\mathbb{C}^{d}}|F(z)|^{2} e^{-\pi|z|^{2}} d z .
$$

A set $\Lambda \subseteq \mathbb{C}^{d}$ is called a sampling set for $\mathcal{F}_{d}^{2}$, if, for some constants $A, B>0$,

$$
A\|F\|_{\mathcal{F}_{d}^{2}}^{2} \leq \sum_{\lambda \in \Lambda}|F(\lambda)|^{2} e^{-\pi|\lambda|^{2}} \leq B\|F\|_{\mathcal{F}_{d}^{2}}^{2}, \quad \forall F \in \mathcal{F}_{d}^{2} .
$$

Our second question deals with the spanning properties of time-frequency shifts of the Gaussian function $\phi(x)=\exp \left(-\pi|x|^{2}\right), x \in \mathbb{R}^{d}$, and thus is a problem for functions on $\mathbb{R}^{d}$. Let $\lambda=\xi+i \eta \in \mathbb{C}^{d}$ with $\xi, \eta \in \mathbb{R}^{d}$ and

$$
\left(\pi_{\lambda} \phi\right)(x)=e^{2 i \pi<\eta, x>} e^{-\pi|x-\xi|^{2}}
$$

be the corresponding time-frequency shift of $\phi$ by $\lambda$. Given a discrete set $\Lambda \subseteq \mathbb{C}^{d}$, we denote the set of time-frequency shifts along $\Lambda$ by

$$
\mathcal{G}(\Lambda)=\left\{\pi_{\lambda} \phi: \lambda \in \Lambda\right\},
$$

2010 Mathematics Subject Classification. 42C15, 33C90, 32A30, 94A12.

Key words and phrases. Gabor frame, Gauss function, lattice, Weierstrass sigma-function, interpolating function, entire functions of several variables.

K. G. was supported in part by the project 31887-N32 the Austrian Science Fund (FWF). 
which is usually called a Gabor family. We then say that $\mathcal{G}(\Lambda)$ is a frame for $L^{2}\left(\mathbb{R}^{d}\right)$, if for some constants $A, B>0$

$$
A\|f\|_{L^{2}\left(\mathbb{R}^{d}\right)}^{2} \leq \sum_{\lambda \in \Lambda}\left|\left\langle f, \pi_{\lambda} \phi\right\rangle\right|^{2} \leq B\|f\|_{L^{2}\left(\mathbb{R}^{d}\right)}^{2}, \quad \forall f \in L^{2}\left(\mathbb{R}^{d}\right)
$$

We are interested in the frame property of $\mathcal{G}(\Lambda)$ in $L^{2}\left(\mathbb{R}^{d}\right)$. This is a "real-variable" problem about functions in $L^{2}\left(\mathbb{R}^{d}\right)$.

It is well-known that the Bargmann transform maps the time-frequency shifts $\pi_{\lambda} \phi$ to the normalized reproducing kernel of $\mathcal{F}_{d}^{2}$ [2]. Therefore these two problems are equivalent via the Bargmann transform:

The system $\mathcal{G}(\Lambda)$ forms a frame in $L^{2}\left(\mathbb{R}^{d}\right)$, if and only if $\Lambda$ is sampling in $\mathcal{F}_{d}^{2}$. See Section 2.1 for a detailed description.

In the case of one variable $d=1$ the sampling property in $\mathcal{F}_{1}^{2}$ and frame property of $\mathcal{G}(\Lambda)$ can be completely characterized in terms of the density of $\Lambda$ by the results in [19,29,30]. Precisely, a separated set $\Lambda \subseteq \mathbb{C}$ is a sampling set for $\mathcal{F}_{1}^{2}$, if and only if its (lower) Beurling $D^{-}(\Lambda)$ is greater than 1. See also the discussion in [10,12,17]. In the multivariate case the density condition $D^{-}(\Lambda)>1$ is necessary (see [21,22]), but is far from sufficient. Sufficient conditions in terms of a covering density are given in [5, 21], but they imply a large Beurling density.

For more detailed results, additional arithmetic conditions are required. For this reason we restrict our attention exclusively to lattices. As in [9] we consider only complex lattices. By a complex lattice we understand a lattice of the form

$$
\Lambda=A(\mathbb{Z}+i \mathbb{Z})^{d}=A \mathbb{Z}[i]^{d}
$$

for some $A \in \mathrm{GL}(d, \mathbb{C})$. Throughout we will write $\mathbb{Z}[i]=\mathbb{Z}+i \mathbb{Z}$ for the ring of Gaussian integers in $\mathbb{C}$. Since $\Lambda$ is a discrete subgroup of $\mathbb{C}^{d}$, the corresponding sampling set and the Gabor family $\mathcal{G}(\Lambda)$ possess an additional structure.

To answer the question about Gabor frames over a lattice, one applies the fundamental duality theory [6, 14, 27] (see also Section 2.1 below). One of the key points is relation between the sampling problem in the space $\mathcal{F}_{d}^{2}$ and the uniqueness problem in the space $\mathcal{F}_{d}^{\infty}$, which consists of entire functions $F$ on $\mathbb{C}^{d}$ such that

$$
\|F\|_{\mathcal{F}_{d}^{\infty}}=\sup _{z \in \mathbb{C}^{d}}|F(z)| e^{-\frac{\pi|z|^{2}}{2}}<\infty .
$$

After a suitable reformulation, we see that the construction of Gabor frames is intimately connected to two fundamental problems about entire functions.

(i) Construct an entire function $\sigma_{\Lambda}$ with possibly smallest growth that vanishes on $\Lambda$, which in analogy to the one-dimensional case we call a sigma-type function for $\Lambda$.

(ii) Construct an entire function $\tau_{\Lambda}$ that is interpolating on $\Lambda$, i.e., $\tau_{\Lambda}(\lambda)=\delta_{\lambda, 0}$ for $\lambda \in \Lambda$. 
In dimension $d=1$ the above problems on $\mathbb{Z}[i]$ are solved by the classical Weierstrass $\sigma$-function

$$
\sigma(z)=z \prod_{m, n \in \mathbb{Z},}\left(1-\frac{z}{m+i n}\right) e^{\frac{z}{m+i n}+\frac{1}{2} \frac{z^{2}}{(m+i n)^{2}}}, \quad \tau(z)=\frac{\sigma(z)}{z} .
$$

Clearly, $\sigma$ is an entire function with $\mathbb{Z}[i]$ as its zero set, and $\tau$ in interpolating on $\mathbb{Z}[i]$.

By contrast, in several complex variables the above questions are rather unusual. The zero set of an entire function of $d>1$ variables is always an analytic manifold. Although it is possible to extend the construction of the Weierstrass product to obtain entire functions whose zero set is a given analytic hypersurface [15,28], this construction sheds no light on the search of sigma-type functions on a lattice. Only few results about interpolation with discrete sets are known, see, e.g., [23, 24].

Our first contribution is a general recipe for the construction of sigma-type functions and interpolating functions associated to an arbitrary complex lattice. The idea builds essentially on the one-dimensional machinery and yields a special class of sigma-type functions, whose zero set is a union of analytic planes that contain the original lattice. Entire functions on $\mathbb{C}^{2}$ with plane zeros also play an important role in [20]. In principle this idea works for arbitrary dimensions, but we will restrict ourselves to entire functions of two variables.

Our second contribution is the application of the general construction of sigmatype functions to show that certain complex lattices fail to yield sampling sets for $\mathcal{F}_{2}^{2}$. This requires the control of the growth of the sigma-type function so that it is in $\mathcal{F}_{d}^{2}$. For the application to sampling in Bargmann-Fock space the goal is therefore to find sigma-type functions with small growth.

To provide an idea of the main construction, we consider a model example that inspired our general construction.

Let

$$
A=\left(\begin{array}{cc}
1 & 1 / 2 \\
0 & \sqrt{3} / 2
\end{array}\right), \quad \Lambda=A \mathbb{Z}[i]^{2} .
$$

This lattice is the complexification of the usual hexagonal lattice in $\mathbb{R}^{2}$. Our main construction then suggests the following sigma-type function for $\Lambda$ :

$$
\sigma_{\Lambda}\left(z_{1}, z_{2}\right)=\sigma\left(z_{1}\right) \sigma\left(\frac{z_{2}}{\sqrt{3}}-\frac{1}{2}\right) \sigma\left(\frac{z_{2}}{\sqrt{3}}-\frac{i}{2}\right) \sigma\left(\frac{z_{2}}{\sqrt{3}}-\frac{1+i}{2}\right) e^{2 \pi(1-i) z_{2}} .
$$

It is easy to check that $\sigma_{\Lambda}$ vanishes on $\Lambda$ and satisfies the growth estimate $\left|\sigma_{\Lambda}(z)\right| \leq C e^{\pi|z|^{2} / 2}$.

The general characterization of lattice sampling sets (Proposition 2.1) then implies the following result:

$$
\Lambda \text { fails to be a sampling set for } \mathcal{F}_{2}^{2} \text {. }
$$

By contrast, the sigma-type function

$$
\sigma_{2}\left(A^{-1} z\right)=\sigma\left(z_{1}-\frac{z_{2}}{\sqrt{3}}\right) \sigma\left(\frac{2 z_{2}}{\sqrt{3}}\right)
$$


also vanishes on $\Lambda$, but it grows much too fast to be of use in the analysis of $\mathcal{F}_{2}^{2}$.

A small modification of (5) yields the interpolating function

$$
\left.\tau_{\Lambda}\left(z_{1}, z_{2}\right)=\frac{\sigma\left(z_{1}\right)}{z_{1}} \sigma\left(\frac{z_{2}}{\sqrt{3}}-\frac{1}{6}\right) \sigma\left(\frac{z_{2}}{\sqrt{3}}-\frac{i}{6}\right) \sigma\right)\left(\frac{z_{2}}{\sqrt{3}}-\frac{1+i}{6}\right) e^{\pi(1-i) / \sqrt{3} z_{2}} .
$$

Although $\tau_{\Lambda} \notin \mathcal{F}_{2}^{2}$, it satisfies the growth rate $\phi_{\Lambda}(z) \leq C e^{\pi|z|^{2} / 2}$. This suffices to derive a weak Lagrange interpolation formula. See Theorem 5.3 .

This example is rather puzzling. In dimension $d=1$ the hexagonal lattice $A \mathbb{Z}^{2}$ has density $2 / \sqrt{3}>1$ and generates a Gabor frame $\mathcal{G}\left(A \mathbb{Z}^{2}\right)$ with certain optimal features [4,32]. By contrast in dimension $d=2, A \mathbb{Z}[i]^{2}$ fails to generate a Gabor frame and a sampling set for $\mathcal{F}_{2}^{2}$, although it has density $4 / 3>1$.

Currently the investigation of sampling the Bargmann-Fock space on lattices is poorly understood, and still amounts to the investigation of examples and counterexamples. We hope that this article will stir some interest among the experts in several complex variables and that it will inspire a deeper analysis of the problem.

The article is organized as follows. In Section 2 we collect the background material about the connection between sampling in Bargmann-Fock space and Gabor frames, the basic information about the Weierstrass sigma-function, and a normalized representation of lattices by means of Minkowski reduced bases. Section 3 contains the main construction of sigma-type functions and interpolating functions for complex lattices. In Section 4 we use this class of sigma-type functions to show that certain "natural" lattices of density $>1$ fail to be sampling in Bargmann-Fock space. In Section 5 we prove a weak Lagrange interpolation formula for certain lattices of density $\geq 1$.

\section{Sampling, Sigma Functions, and Lattices}

2.1. Sampling in Bargmann-Fock space and Gabor frames. The lattice structure leads to special criteria for a set $\Lambda$ to be sampling. We emphasize that these are unavailable for arbitrary sets of points.

The relation between the sampling property of the lattice $\Lambda$ and the frame property of the system $\mathcal{G}(\Lambda)$ is summarized in the following statement. For its formulation we need the adjoint lattice $\Lambda^{\circ}=\left(A^{*}\right)^{-1} \mathbb{Z}[i]^{d}$ of $\Lambda=A \mathbb{Z}[i]^{d}$

Proposition 2.1. For a lattice $\Lambda=A \mathbb{Z}[i]^{d} \subseteq \mathbb{C}^{d}$ the following are equivalent:

(i) $\mathcal{G}(\Lambda)$ is a frame in $L^{2}\left(\mathbb{R}^{d}\right)$;

(ii) $\Lambda$ is a sampling set for $\mathcal{F}_{d}^{2}$;

(iii) There exists an interpolating function $G \in \mathcal{F}_{d}^{2}$ for $\Lambda^{\circ}$ satisfying the Bessel property, i.e.,

$$
G(\mu)=\delta_{\mu, 0}, \quad \text { for all } \mu \in \Lambda^{\circ},
$$

and $F \rightarrow\left(\left\langle F, e^{\pi \bar{\lambda} \cdot z} G(z)\right\rangle e^{-\pi|\lambda|^{2} / 2}\right)_{\lambda \in \Lambda}$ maps $\mathcal{F}_{d}^{2}$ to $\ell^{2}(\Lambda)$

(iv) $\Lambda$ is a set of uniqueness for $\mathcal{F}_{d}^{\infty}$, i.e., if $F \in \mathcal{F}_{d}^{\infty}$ and $F(\lambda)=0$ for all $\lambda \in \Lambda$, then $F \equiv 0$. 
Proposition 2.1 explains the fundamental importance of sigma-type functions and interpolating functions in $\mathcal{F}_{d}^{2}$ for the theory of Gabor frames. It follows from this proposition that a function $F \in \mathcal{F}_{d}^{2}$ can be recovered from its samples $\{F(\lambda)\}_{\lambda \in \Lambda}$ by mean of the interpolating function $G$ constructed for $\Lambda^{\circ}$, not for $\Lambda$ ! Namely 1

$$
F(z)=\sum_{\lambda \in \Lambda} F(\lambda) e^{\pi \bar{\lambda} \cdot z} G(z-\lambda) e^{-\pi|\lambda|^{2} / 2} .
$$

The connection (i) and (ii) is classical. Let $f \in L^{2}\left(\mathbb{R}^{d}\right)$ and

$$
B f(z)=2^{d / 4} e^{-\pi z \cdot z / 2} \int_{\mathbb{R}^{d}} f(x) e^{-\pi|x|^{2}+2 \pi x \cdot z} d x
$$

be the Bargmann transform of $f$. Then $B$ is unitary from $L^{2}\left(\mathbb{R}^{d}\right)$ onto the BargmannFock space $\mathcal{F}_{d}^{2}$, e.g., by [8]. Moreover, the Bargmann transform maps the timefrequency shift $2^{d / 4} \pi_{\lambda} \phi$ to the normalized reproducing kernel $e^{\pi \bar{\lambda} \cdot z} e^{-\pi|\lambda|^{2} / 2}$ of the Fock space. Therefore

$$
\left\langle f, \pi_{\lambda} \phi\right\rangle_{L^{2}}=\left\langle B f, B\left(\pi_{\lambda} \phi\right)\right\rangle_{\mathcal{F}_{d}^{2}}=B f(\lambda) e^{-\pi|\lambda|^{2} / 2} .
$$

See [8,10] and the original literature [2].

The equivalence of (ii) and (iii) seems beyond the realm of complex analysis and is due to the invariance properties of lattices. It shows that the problem of sampling on a given lattice $\Lambda$ is equivalent to an interpolation problem of the adjoint lattice $\Lambda^{\circ}$. This statement is part of the duality theory of Gabor frames [6, 14, 27].

The equivalence of (ii) and (iv) follows from one of the characterizations of Gabor frames without inequalitites [11] via the Bargmann transform.

2.2. Sigma-type functions for lattices. Our main tool for the construction of sigma-type functions in $\mathbb{C}^{d}$ is the classical Weierstrass $\sigma$-function of one variable

$$
\sigma(z)=z \prod_{\lambda \in \mathbb{Z}[i]}\left(1-\frac{z}{\lambda}\right) e^{\frac{z}{\lambda}+\frac{1}{2} \frac{z^{2}}{\lambda^{2}}}
$$

We refer to [1,31] for basic properties of $\sigma(z)$. In particular, $\sigma$ is an entire function with $\mathbb{Z}[i]$ as the zero set.

In addition, see e.g. [16], for each $\epsilon>0$

$$
|\sigma(z)| \asymp e^{\pi|z|^{2} / 2}, \quad \operatorname{dist}(z, \mathbb{Z}[i])>\epsilon .
$$

We note that $\sigma \in \mathcal{F}_{1}^{\infty} \backslash \mathcal{F}_{1}^{2}$. By Proposition 2.1 the lattice of Gaussian integers $\mathbb{Z}[i]$ fails to be a sampling set for $\mathbb{Z}[i]$. This fact was already proved in [3, 25]

For $z=\left(z_{1}, \ldots, z_{d}\right) \in \mathbb{C}^{d}$ we set

$$
\sigma_{0}(z)=\prod_{j=1}^{d} \sigma\left(z_{j}\right) .
$$

Then the function

$$
\sigma_{A}(z)=\sigma_{0}\left(A^{-1} z\right)
$$

\footnotetext{
${ }^{1}$ We always use the "real" inner product: $z \cdot w=\sum_{j=1}^{d} z_{j} w_{j}$ for $z=\left(z_{j}\right), w=\left(w_{j}\right) \in \mathbb{C}^{d}$.
} 
vanishes on $\Lambda$ and satisfies the growth estimate

$$
\left|\sigma_{A}(z)\right| \leq C e^{\pi\left\|A^{-1}\right\|_{o p}^{2}|z|^{2} / 2},
$$

where as usual $\|A\|_{o p}$ denotes the largest singular value of $A$.

Similarly we construct the interpolating function for $\Lambda=A \mathbf{Z}^{d}$. We define $\tau(z)=z^{-1} \sigma(z)$ for $z \in \mathbb{C}$ and

$$
\tau_{0}(z)=\prod_{j=1}^{d} \tau\left(z_{j}\right) ; \tau_{\Lambda}(z)=\tau_{0}\left(A^{-1} z\right) \text { for } z=\left(z_{1}, \ldots, z_{d}\right) \in \mathbb{C}^{d} .
$$

For example, consider the lattice $\alpha \mathbb{Z}[i] \subseteq \mathbb{C}$ with adjoint lattice $\Lambda^{\circ}=\alpha^{-1} \mathbb{Z}[i]$. Then $\Lambda^{\circ}$ possesses the interpolating function $\tau(\alpha z)$, which belongs to $\mathcal{F}_{1}^{2}$, if and only if $0<|\alpha|<1$. By Proposition $2.1 \alpha \mathbb{Z}[i]$ is a sampling set for $\mathcal{F}_{1}^{2}$, if and only if $|\alpha|<1$. This is a baby version of the complete characterization of one-dimensional sampling sets in [19,29,30].

2.3. Lattice reduction and Minkowski-reduced bases. Clearly, the functions $\sigma_{\Lambda}$ and $\tau_{\Lambda}$ depend on the generating matrix $A$, or equivalently, on the choice of a basis of $\Lambda$. Our first task is to choose a suitable basis of $\Lambda$, so that $\left\|A^{-1}\right\|_{\text {op }}$ is small. One of possible recipes is to choose a basis of $\Lambda$ which consists of the shortest possible vectors.

Precisely, choose vectors $\mathbf{a}_{j} \in \Lambda$, such that $\left\|\mathbf{a}_{1}\right\|=\min \{\|\lambda\|: \lambda \in \Lambda\}$ and

$$
\left\|\mathbf{a}_{j}\right\|=\min \left\{\|\lambda\|: \lambda \in \Lambda, \lambda \notin \operatorname{span}\left[\mathbf{a}_{1}, \ldots \mathbf{a}_{j-1}\right]\right\} .
$$

Such a basis is called Minkowski reduced for $\Lambda$ [18], and satisfies

$$
\left\|\mathbf{a}_{1}\right\| \leq\left\|\mathbf{a}_{2}\right\| \leq \cdots \leq\left\|\mathbf{a}_{d}\right\| .
$$

After setting $A=\left(\begin{array}{llll}\mathbf{a}_{1} & \mathbf{a}_{2} & \ldots & \mathbf{a}_{d}\end{array}\right)$, the lattice is $\Lambda=A \mathbb{Z}[i]^{d}$.

We may write $A=U S$, where $U$ is a unitary matrix and $S$ is an upper triangular matrix with real values on the diagonal and with columns $\mathbf{b}_{j}$ (by QR-factorization or Gram-Schmidt orthogonalization). Since the Fock space $\mathcal{F}_{d}^{2}$ is invariant under a unitary transformation $U$ of coordinates, a set $\Lambda$ is sampling, if and only if $U^{-1} \Lambda$ is sampling. Replacing $\Lambda=A \mathbb{Z}[i]^{d}$ by $U^{-1} \Lambda=S \mathbb{Z}[i]^{d}$, we may therefore assume without loss of generality that $\Lambda=S \mathbb{Z}[i]^{d}$ for an upper triangular matrix $S$.

Since $U$ preserves lengths, the columns of $S$ are also Minkowski-reduced basis, and $\left\|\mathbf{b}_{1}\right\| \leq\left\|\mathbf{b}_{2}\right\| \leq \cdots \leq\left\|\mathbf{b}_{d}\right\|$. The upper triangular matrix $S$ is of the form

$$
S=\left(s_{j k}\right)=\left(\begin{array}{cccc}
\gamma_{1} & * & \ldots & * \\
0 & \gamma_{2} & \ldots & * \\
\vdots & & & \\
0 & \ldots & 0 & \gamma_{d}
\end{array}\right) \text {, }
$$

and its entries satisfy the additional conditions

$$
\begin{aligned}
& s_{j, k}=0 \quad k<j, \quad s_{k k}=\gamma_{k}>0, \\
& \left|\operatorname{Re} s_{j, k}\right|, \quad\left|\operatorname{Im} s_{j, k}\right| \leq \frac{s_{j, j}}{2} .
\end{aligned}
$$


In [9] we proved the following result for general upper-diagonal matrices in arbitrary dimension.

Proposition 2.2. Let $\Lambda=S \mathbb{Z}[i]^{d}$ with an upper triangular matrix $S$.

(i) If $\gamma_{j}<1$ for $j=1, \ldots, d$, then $\Lambda$ is a sampling set for $\mathcal{F}_{d}^{2}$ (and $\mathcal{G}(\Lambda)$ is a frame for $L^{2}\left(\mathbb{R}^{d}\right)$ ).

(ii) If $\gamma_{d} \geq 1$, then $\Lambda$ is not sampling and $\mathcal{G}(\Lambda)$ is not a frame.

The proof goes roughly as follows. The adjoint lattice is $\Lambda^{\circ}=\left(S^{*}\right)^{-1} \mathbb{Z}[i]^{d}$, where $\left(S^{*}\right)^{-1}$ is a lower trigonal matrix with diagonal $\left(\lambda_{j}^{-1}\right)_{j=1, \ldots, d}$. Then the function

$$
F_{0}\left(z_{1}, z_{2}, \ldots, z_{d}\right)=\prod_{j=1}^{d} \frac{\sigma\left(\gamma_{j} z_{j}\right)}{z_{j}}
$$

belongs to $\mathcal{F}_{d}^{2}$ and solves the interpolation problem (iii) in Proposition 2.1. If $\gamma_{d} \geq 1$, then the function $F_{1}\left(z_{1}, z_{2}, \ldots, z_{d}\right):=\sigma\left(\gamma_{d}^{-1} z_{d}\right)$ belongs to $\mathcal{F}_{d}^{\infty}$ and vanishes on $\Lambda$. By Proposition 2.1 $\Lambda$ fails to be a sampling set.

A different class of examples is discussed in [26].

From now on we deal with explicit constructions in two complex variables. We may assume without loss of generality that $\Lambda$ is determined by the matrix

$$
A=\left(\begin{array}{cc}
\gamma_{1} & \beta \\
0 & \gamma_{2}
\end{array}\right)
$$

with

$$
\gamma_{1}, \gamma_{2}>0, \quad \gamma_{1}^{2} \leq|\beta|^{2}+\gamma_{2}^{2}
$$

Since the basis is reduced, we have

$$
|\operatorname{Re} \beta|, \quad|\operatorname{Im} \beta| \leq \frac{\gamma_{1}}{2} .
$$

\section{A Construction of Sigma-Type Functions and Interpolating Functions via Sublattices}

To go beyond Proposition 2.2, we need a more involved recipe for sigma-type and interpolating functions. In this section we provide such a general construction inspired by the model example (5). The goal is to produce sigma-type functions or interpolation functions with small growth.

Given a lattice $\Lambda=A \mathbb{Z}[i]^{2}$ in $\mathbb{C}^{2}$, we will use the following master plan to construct a sigma-type function function or an interpolating function on $\Lambda$ via a sublattice.

(1) Construct a sublattice $\Gamma \subseteq \Lambda$ that possesses an orthogonal basis (or possibly a nearly orthogonal basis).

(2) Construct a sigma function $\sigma_{\Gamma}$ and an interpolating function $\tau_{\Gamma}$ on $\Gamma$ according to (13) and (15).

(3) Determine a suitable set of coset representatives $\Lambda / \Gamma$.

(4) The sigma function and the interpolating function on $\Lambda$ will be a suitable product of shifts of each of the factors of $\sigma_{\Gamma}$ and $\tau_{\Gamma}$. 
3.1. Sublattices and their cosets. Following the outline above, we first describe sublattices and their cosets in $\mathbb{C}^{2}$.

Every (complex) sublattice of $\mathbb{Z}[i]^{2}$ is of the form $B \mathbb{Z}[i]^{2}$ for an invertible matrix $2 \times 2$-matrix $B$ with entries in $\mathbb{Z}[i]$ (in short, $B \in \mathrm{GL}(2, \mathbb{Z}[i])$ ). Consequently, every sublattice of a lattice $\Lambda=A \mathbb{Z}[i]^{2} \subseteq \mathbb{C}^{2}$ is of the form

$$
\Gamma=A B \mathbb{Z}[i]^{2}
$$

for some $B \in \mathrm{GL}(2, \mathbb{Z}[i])$.

Clearly, we can represent the full lattice $\Lambda$ as a union of shifts of the sublattice $\Gamma$ as follows $\Lambda=\bigcup_{j=1}^{n}\left(\delta_{j}+\Gamma\right)$ for some lattice points $\delta_{j} \in \Lambda$. In fact, the shifts $\delta_{j}$ are the representatives of the quotient $\Lambda / \Gamma$. We will use the following explicit parametrization of $\mathbb{Z}[i]^{2} / B \mathbb{Z}[i]^{2}$ and hence of $\Lambda / \Gamma$.

Lemma 3.1. Let $B=\left(\begin{array}{ll}a & c \\ b & d\end{array}\right)$ with entries $a, b, c, d \in \mathbb{Z}[i]$. Let $\gamma=\operatorname{gcd}(a, c) \in \mathbb{Z}[i]$ 2 and $\Delta=\operatorname{det} B=a d-b c$. Let $Q=([0,1)+i[0,1)) \subseteq \mathbb{C}$. Then the set

$$
\mathcal{D}=\left\{\left(\delta_{1}, \delta_{2}\right) \in \mathbb{Z}[i]^{2}: \delta_{1} \in \gamma Q \cap \mathbb{Z}[i], \delta_{2} \in \frac{\Delta}{\gamma} Q \cap \mathbb{Z}[i]\right\}
$$

is a set of coset representatives for $\mathbb{Z}[i]^{2} / B \mathbb{Z}[i]^{2}$.

In particular, if $B$ possesses real-valued entries $a, b, c, d \in \mathbb{Z}$, and $a$ and $c$ are relatively prime over $\mathbb{Z}$, then

$$
\left.\mathcal{D}=\left\{(0, \delta) ; \delta=\alpha+i \alpha^{\prime}\right), 0 \leq \alpha, \alpha^{\prime}<|\operatorname{det} B|\right\}
$$

is a set of coset representatives of $\mathbb{Z}[i]^{2} / B \mathbb{Z}[i]^{2}$.

Proof. We observe that the set $Q$ is a (half-open) square in $\mathbb{C}$ and that $(Q-$ $Q) \cap \mathbb{Z}[i]=\{0\}$, where $Q-Q=(-1,1)+i(-1,1)$ is the difference set. We write $a=\gamma a^{\prime}, c=\gamma c^{\prime}$ for $a^{\prime}, c^{\prime} \in \mathbb{Z}[i]$, and note that $\Delta=\gamma\left(a^{\prime} d-b c^{\prime}\right)$, so that $\Delta / \gamma \in \mathbb{Z}[i]$.

Assume that $\delta_{1}, \delta_{1}^{\prime} \in \gamma Q \cap \mathbb{Z}[i], \delta_{2}, \delta_{2}^{\prime} \in \frac{\Delta}{\gamma} Q \cap \mathbb{Z}[i]$ and that $\left(\delta_{1}-\delta_{1}^{\prime}, \delta_{2}-\delta_{2}^{\prime}\right)^{T}=$ $B(k, l)^{T}$ for some $k, l \in \mathbb{Z}[i]$, in other words $\left(\delta_{1}, \delta_{1}^{\prime}\right)$ and $\left(\delta_{2}, \delta_{2}^{\prime}\right)$ represent the same coset. Then

$$
\left(\begin{array}{ll}
a & c \\
b & d
\end{array}\right)\left(\begin{array}{l}
k \\
l
\end{array}\right)=\left(\begin{array}{l}
a k+c l \\
b k+d l
\end{array}\right)=\left(\begin{array}{l}
\delta_{1}-\delta_{1}^{\prime} \\
\delta_{2}-\delta_{2}^{\prime}
\end{array}\right) .
$$

The first coordinate is

$$
\delta_{1}-\delta_{1}^{\prime}=a k+c l=\gamma\left(a^{\prime} k+c^{\prime} l\right) \in \gamma(Q-Q) \cap \mathbb{Z}[i],
$$

and therefore the Gaussian integer $a^{\prime} k+c^{\prime} l$ is in $Q-Q$, which implies that $a^{\prime} k+$ $c^{\prime} l=0$. Since $\operatorname{gcd}\left(a^{\prime}, c^{\prime}\right)=1$ (up to multiplication by $\pm 1, \pm i$ ), it follows that $l=N a^{\prime}, k=-N c^{\prime}$ for some $N \in \mathbb{Z}[i]$.

Now the second coordinate of $B(k, l)^{T}$ is

$$
b k+d l=N\left(-b c^{\prime}+a^{\prime} d\right)=N \frac{\Delta}{\gamma} \in \frac{\Delta}{\gamma}(Q-Q) \cap \mathbb{Z}[i] .
$$

Therefore $N \in(Q-Q) \cap(\mathbb{Z}[i])$. This implies that $N=0$ and thus $k=l=0$. Altogether we have shown that $\left(\delta_{1}, \delta_{1}^{\prime}\right)=\left(\delta_{2}, \delta_{2}^{\prime}\right)$. It is easily verified that the

\footnotetext{
${ }^{2}$ Note that over $\mathbb{Z}[i]$ the greatest common divisor is only determined up to multiplication with $\pm 1, \pm i$. We refer the reader to [13] for the facts on division in $\mathbb{Z}[i]$.
} 
cardinality of $\gamma Q \cap \mathbb{Z}[i]$ is $|\gamma|^{2}$ for $\gamma \in \mathbb{Z}[i]$, therefore the cardinality of $\mathcal{D}$ is $|\operatorname{det} B|^{2}$. Consequently $\mathcal{D}$ is a complete set of representatives of $\mathbb{Z}[i]^{2} / B \mathbb{Z}[i]^{2}$.

3.2. A construction of sigma-type functions. We begin with the calculation of $\sigma_{\Gamma}$ and $\tau_{\Gamma}$ on the cosets $\nu+\Gamma$ of $\Gamma$ in $\Lambda$. Let $B=\left(\begin{array}{cc}a & c \\ b & d\end{array}\right), \Delta=\operatorname{det} B$ and $\Gamma=A B \mathbb{Z}[i]^{2}$ be the sublattice of $\Lambda=A \mathbb{Z}[i]^{2}$. In view of the explicit examples, we assume furthermore that $|\operatorname{gcd}(a, c)|=1$.

Let, as before, $\sigma(z)$ be the classical Weierstrass $\sigma$-function for $\mathbb{Z}[i]$. For $z=$ $\left(z_{1}, z_{2}\right) \in \mathbb{C}$ we denote $\sigma \otimes \sigma(z)=\sigma\left(z_{1}\right) \sigma\left(z_{2}\right)$ and set

$$
\sigma_{\Gamma}(z)=(\sigma \otimes \sigma)\left((A B)^{-1} z\right) .
$$

We first evaluate $\sigma_{\Gamma}$ on $\Lambda$. Since $|\operatorname{gcd}(a, c)|=1$, the representatives of $\mathbb{Z}[i]^{2} / B \mathbb{Z}[i]^{2}$ can be chosen to be $(0, \delta)$, and thus a general lattice point $\lambda \in \Lambda=A \mathbb{Z}[i]^{2}$ can be written as

$$
\lambda=A\left(B\left(\begin{array}{l}
k \\
l
\end{array}\right)+\left(\begin{array}{l}
0 \\
\delta
\end{array}\right)\right)
$$

for some $k, l \in \mathbb{Z}[i]$ and $(0, \delta) \in \mathcal{D}$. Consequently

$$
\begin{aligned}
\sigma_{\Gamma}(\lambda) & =(\sigma \otimes \sigma)\left((A B)^{-1}\left(A B(k, l)^{T}+A(0, \delta)^{T}\right)\right) \\
& =(\sigma \otimes \sigma)\left(\left(\begin{array}{l}
k \\
l
\end{array}\right)+B^{-1}\left(\begin{array}{l}
0 \\
\delta
\end{array}\right)\right) .
\end{aligned}
$$

Since

$$
B^{-1}\left(\begin{array}{l}
0 \\
\delta
\end{array}\right)=\frac{1}{\Delta}\left(\begin{array}{cc}
d & -c \\
-b & a
\end{array}\right)\left(\begin{array}{l}
0 \\
\delta
\end{array}\right)=\left(\begin{array}{c}
-c \delta / \Delta \\
a \delta / \Delta
\end{array}\right),
$$

the sigma function of the sublattice $\Gamma$ evaluated on $\Lambda$ is

$$
\sigma_{\Gamma}(\lambda)=\sigma\left(k-\frac{c \delta}{\Delta}\right) \sigma\left(l+\frac{a \delta}{\Delta}\right) .
$$

Here is our key observation:

If $\frac{\Delta}{c}$ divides $\delta$, then $\frac{c \delta}{\Delta} \in \mathbb{Z}[i]$ and $\sigma\left(k-\frac{c \delta}{\Delta}\right)=0$, thus $\sigma_{\Gamma}\left(\left(\begin{array}{l}0 \\ \delta\end{array}\right)+\gamma\right)=0$ for all $\gamma \in \Gamma$.

Similarly, if $\frac{\Delta}{a}$ divides $\delta$, then $\sigma(l+a \delta / \Delta)=0$. Thus a single factor of $\sigma_{\Gamma}$ in the product (24) vanishes on the whole coset $(0, \delta)+\Gamma$ and we need not include additional factors to $\sigma_{\Lambda}$ in order to annihilate this coset. We will therefore try to choose a sublattice $\Gamma$ such that $a$ or $c$ divides $\operatorname{det} B=\Delta$ and $\Delta / c$ is small.

To take care of the cosets that do not vanish in this way, we use (one-dimensional) Fock shifts. For $z, \zeta \in \mathbb{C}$ let

$$
\beta_{\zeta} f(z)=e^{\pi \bar{\zeta} z-\pi|\zeta|^{2} / 2} f(z-\zeta) .
$$

We have

$$
e^{-\frac{\pi}{2}|z|^{2}}\left|\beta_{\zeta} f(z)\right|=e^{-\frac{\pi}{2}|z-\zeta|^{2}}|f(z-\zeta)|
$$


so $\beta_{\zeta}$ is a unitary operator on $\mathcal{F}_{1}^{2}$ and an isometry on $\mathcal{F}_{1}^{\infty}$. In particular, together with (11) this yields

$$
\left|\beta_{\zeta} \sigma(z)\right| \asymp e^{\pi|z|^{2} / 2}, \text { if } \operatorname{dist}(z, \mathbb{Z}[i]+\zeta)>\epsilon .
$$

For $z=\left(z_{1}, z_{2}\right) \in \mathbb{C}^{2}$ let $p_{1}\left(z_{1}, z_{2}\right)=z_{1}$ and $p_{2}\left(z_{1}, z_{2}\right)=z_{2}$ be the projections onto the first and second coordinate of $z$. In this notation $\sigma_{\Gamma}(z)=$ $\sigma\left(p_{1}\left((A B)^{-1} z\right)\right) \sigma\left(p_{2}\left((A B)^{-1} z\right)\right)$.

We split the construction of a sigma-type function for $\Lambda$ into several steps.

Step 1. Partition the coset representatives $\mathcal{D}$ into disjoint subsets

$$
\mathcal{D}=\left\{(0, \delta) \in \mathcal{D}: \frac{\Delta}{c} \mid \delta\right\} \cup \mathcal{E}_{1} \cup \mathcal{E}_{2}=\mathcal{E}_{0} \cup \mathcal{E}_{1} \cup \mathcal{E}_{2},
$$

or

$$
\mathcal{D}=\left\{(0, \delta) \in \mathcal{D}: \frac{\Delta}{a} \mid \delta\right\} \cup \mathcal{E}_{1}^{\prime} \cup \mathcal{E}_{2}^{\prime} .
$$

where $\mathcal{E}_{1}$ and $\mathcal{E}_{2}$ are a convenient or arbitrary partition of those $\delta$ with $\frac{\Delta}{c} \not \delta$.

Step 2. We now define the entire functions

$$
\begin{gathered}
\sigma_{\Lambda}(z)= \\
=\sigma\left(p_{1}\left((A B)^{-1} z\right)\right) \prod_{\nu \in \mathcal{E}_{1}}\left(\beta_{\left.p_{1}\left(B^{-1} \nu\right)\right)} \sigma\right)\left(p_{1}\left((A B)^{-1} z\right)\right) \prod_{\nu \in \mathcal{E}_{2}}\left(\beta_{p_{2}\left(B^{-1} \nu\right)} \sigma\right)\left(p_{2}\left((A B)^{-1} z\right)\right),
\end{gathered}
$$

and

$$
\begin{gathered}
\tilde{\sigma}_{\Lambda}(z)= \\
=\sigma\left(p_{2}\left((A B)^{-1} z\right)\right) \prod_{\nu \in \mathcal{E}_{1}^{\prime}}\left(\beta_{\left.p_{1}\left(B^{-1} \nu\right)\right)} \sigma\right)\left(p_{1}\left((A B)^{-1} z\right)\right) \prod_{\nu \in \mathcal{E}_{2}^{\prime}}\left(\beta_{p_{2}\left(B^{-1} \nu\right)} \sigma\right)\left(p_{2}\left((A B)^{-1} z\right)\right) .
\end{gathered}
$$

Proposition 3.2. The functions $\sigma_{\Lambda}$ and $\tilde{\sigma}_{\Lambda}$ vanish on $\Lambda$.

Proof. Let $k, l \in \mathbb{Z}[i],(0, \delta) \in \mathcal{D}, \nu=(0, \eta) \in \mathcal{D}$ and let $\lambda=A\left(B\left(\begin{array}{l}k \\ l\end{array}\right)+\left(\begin{array}{l}0 \\ \delta\end{array}\right)\right)$ be a general lattice point in $\Lambda$. We write the coordinates of the argument in (24) as

$$
p_{1}\left((A B)^{-1} \lambda\right)=k-c \delta / \Delta \quad \text { and } \quad p_{2}\left((A B)^{-1} \lambda\right)=l+a \delta / \Delta .
$$

If we omit the normalizing factors of the Fock shifts, we have

$$
\begin{aligned}
& \beta_{p_{1}\left(B^{-1} \nu\right)} \sigma\left(p_{1}\left((A B)^{-1} \lambda\right) \asymp \sigma\left(k-\frac{c \delta}{\Delta}+\frac{c \eta}{\Delta}\right),\right. \\
& \beta_{p_{2}\left(B^{-1} \nu\right)} \sigma\left(p_{2}\left((A B)^{-1} \lambda\right) \asymp \sigma\left(l+\frac{a \delta}{\Delta}-\frac{a \eta}{\Delta}\right) .\right.
\end{aligned}
$$

If $(0, \delta) \in \mathcal{E}_{0}$, i.e., $\frac{\Delta}{c} \mid \delta$ and thus $\delta=r \frac{\Delta}{c}$ for some $r \in \mathbb{Z}[i]$, then

$$
\sigma\left(p_{1}\left((A B)^{-1} \lambda\right)\right) \asymp \sigma(k-c \delta / \Delta)=\sigma(k-r)=0 .
$$

So $\sigma$ vanishes on the cosets $(0, \delta)+\Gamma$, whenever $\frac{\Delta}{c} \mid \delta$. 
If $(0, \delta) \in \mathcal{E}_{1}$, i.e., $\frac{\Delta}{c} \not \delta$, then the factor

$$
\beta_{p_{1}\left(B^{-1} \nu\right)} \sigma\left(p_{1}\left((A B)^{-1} \lambda\right) \asymp \sigma\left(k-\frac{c \delta}{\Delta}+\frac{c \eta}{\Delta}\right)\right.
$$

vanishes for $\nu=(0, \delta) \in \mathcal{E}_{1}$. Likewise, if $(0, \delta) \in \mathcal{E}_{2}$, then for $\nu \in \mathcal{E}_{2}$

$$
\beta_{p_{2}\left(B^{-1} \nu\right)} \sigma\left(p_{2}\left((A B)^{-1} \lambda\right) \asymp \sigma\left(l+\frac{a \delta}{\Delta}-\frac{a \eta}{\Delta}\right)=0 .\right.
$$

Thus every coset $(0, \delta)^{T}+\Gamma$ is annihilated by a single factor of $\sigma_{\Lambda}$, and $\sigma_{\Lambda}$ vanishes on $\Lambda$.

The proof for $\tilde{\sigma}_{\Lambda}$ is the same.

3.3. Interpolating Functions. For the interpolating function on $\Lambda$ we make a similar ansatz. However, we need to pay special attention to the cosets in $\mathcal{E}_{0}$, where $\frac{\Delta}{c} \mid \delta$. We partition $\mathcal{D}=\mathcal{E}_{0} \cup \mathcal{E}_{1} \cup \mathcal{E}_{2}$ as in (27). Evaluating $p_{j}\left((A B)^{-1} \lambda\right), j=1,2$, on these cosets, eventually leads to the following definitions. Let $b_{1}, b_{2}$ be the basis vectors of $\Gamma$, namely $b_{1}=A B\left(\begin{array}{l}1 \\ 0\end{array}\right)$ and $b_{2}=A B\left(\begin{array}{l}0 \\ 1\end{array}\right)$, and set

$$
b_{3}=b_{2}+A\left(\begin{array}{c}
0 \\
\Delta / c
\end{array}\right) \in \mathbb{C}^{2} \text { and } \zeta=\frac{\left\langle b_{3}, b_{2}\right\rangle}{\left\|b_{2}\right\|^{2}} \in \mathbb{C} .
$$

Recall that $\tau(w)=\sigma(w) / w, w \in \mathbb{C}$ is interpolating for $\mathbb{Z}[i]$ and define the entire function

$$
\begin{aligned}
\tau_{\Lambda}(z)=\tau & \left(p_{1}\left((A B)^{-1} z\right)\right) \prod_{\nu \in \mathcal{E}_{1}}\left(\beta_{p_{1}\left(B^{-1} \nu\right)} \sigma\right)\left(p_{1}\left((A B)^{-1} z\right)\right) \\
& \times \prod_{\nu \in \mathcal{E}_{2}}\left(\beta_{p_{2}\left(B^{-1} \nu\right)} \sigma\right)\left(p_{2}\left((A B)^{-1} z\right)\right) \prod_{r=c \delta / \Delta \in \mathbb{Z}[i] \backslash\{0\}} \beta_{r \zeta} \sigma\left(\frac{\left\langle z, b_{2}\right\rangle}{\left\|b_{2}\right\|^{2}}\right) .
\end{aligned}
$$

The construction of $\tau_{\Lambda}$ is more subtle, since we need to include a finite number of extra factors $\beta_{r \zeta} \sigma\left(\frac{\left\langle z, b_{2}\right\rangle}{\left\|b_{2}\right\|^{2}}\right)$.

Proposition 3.3. $\tau_{\Lambda}$ is an interpolating function for $\Lambda$.

Proof. The proof is similar to the proof of Proposition 3.2. Let $(0, \delta) \in \mathcal{D}, \nu=$ $(0, \eta) \in \mathcal{D}$ and $\lambda=A\left(B\left(\begin{array}{l}k \\ l\end{array}\right)+\left(\begin{array}{l}0 \\ \delta\end{array}\right)\right)$ be a general lattice point in $\Lambda$. Then as before we have

$$
\begin{aligned}
\tau\left(p_{1}\left((A B)^{-1} \lambda\right)\right) & =\tau\left(k-\frac{c \delta}{\Delta}\right) \\
\beta_{p_{1}\left(B^{-1} \nu\right)} \sigma\left(p_{1}\left((A B)^{-1} \lambda\right)\right) & \asymp \sigma\left(k-\frac{c \delta}{\Delta}+\frac{c \eta}{\Delta}\right) \\
\beta_{p_{2}\left(B^{-1} \nu\right)} \sigma\left(p_{2}\left((A B)^{-1} \lambda\right)\right) & \asymp \sigma\left(l+\frac{a \delta}{\Delta}-\frac{a \eta}{\Delta}\right) .
\end{aligned}
$$

If $\lambda=0(k=l=\delta=0)$, then

$$
\tau_{\Lambda}(0) \asymp \tau(0) \prod_{\eta \in p_{1}\left(\mathcal{E}_{1}\right)} \sigma\left(\frac{c \eta}{\Delta}\right) \prod_{\eta \in p_{2}\left(\mathcal{E}_{2}\right)} \sigma\left(\frac{-a \eta}{\Delta}\right) \neq 0
$$


since by definition $\tau(0)=1$ and $\frac{c \eta}{\Delta} \notin \mathbb{Z}[i], \frac{-a \eta}{\Delta} \notin \mathbb{Z}[i]$ for $\nu \in \mathcal{E}_{1} \cup \mathcal{E}_{2}$.

If $(0, \delta) \in \mathcal{E}_{1} \cup \mathcal{E}_{2}$, then $\tau_{\Lambda}(\lambda)=0$ as in Proposition 3.2 .

Finally, if $(0, \delta) \in \mathcal{E}_{0}$, then $\delta=r \Delta / c$ for some $r \in \mathbb{Z}[i]$. Therefore

$$
\tau\left(p_{1}\left((A B)^{-1} z\right)\right)=\tau\left(k-\frac{c \delta}{\Delta}\right)=\tau(k-r)
$$

and $\tau_{\Lambda}(\lambda)=0$, unless $k=r$.

If $k=r$ or $\delta=r \Delta / c$, then, with the notation of (29),

$$
\lambda=A\left(B\left(\begin{array}{l}
r \\
l
\end{array}\right)+\left(\begin{array}{c}
0 \\
r \Delta / c
\end{array}\right)\right)=l A B\left(\begin{array}{l}
0 \\
1
\end{array}\right)+r\left(A B\left(\begin{array}{l}
1 \\
0
\end{array}\right)+A\left(\begin{array}{c}
0 \\
\Delta / c
\end{array}\right)\right)=l b_{2}+r b_{3} .
$$

We consider the factor $\beta_{r \zeta} \sigma\left(\frac{\left\langle z, b_{2}\right\rangle}{\left\|b_{2}\right\|^{2}}\right)$ of $\tau_{\Lambda}$ with $\zeta=\frac{\left\langle b_{3}, b_{2}\right\rangle}{\left\|b_{2}\right\|^{2}}$ and evaluate at $\lambda$ :

$$
\begin{aligned}
\beta_{r \zeta} \sigma\left(\frac{\left\langle\lambda, b_{2}\right\rangle}{\left\|b_{2}\right\|^{2}}\right) & \asymp \sigma\left(\frac{\left\langle\lambda, b_{2}\right\rangle}{\left\|b_{2}\right\|^{2}}-r \zeta\right) \\
& =\sigma\left(\frac{\left\langle l b_{2}+r b_{3}, b_{2}\right\rangle}{\left\|b_{2}\right\|^{2}}-r \frac{\left\langle b_{3}, b_{2}\right\rangle}{\left\|b_{2}\right\|^{2}}\right)=\sigma(l)=0 .
\end{aligned}
$$

Altogether we have shown that $\tau_{\Lambda}(\lambda)=\tau_{\lambda}(0) \delta_{\lambda, 0}$ and that $\tau_{\Lambda}(0) \neq 0$, so that $\tau_{\Lambda}$ is an interpolating function for $\Lambda$.

\section{Failure of Sampling in Fock Space}

We now apply the construction of sigma-type functions to prove the failure of sampling for certain lattices of density $>1$. Such examples appear naturally if one considers a sigma-type function of sufficiently small growth. Its zero set is a "lattice" of hyperplanes, and one can then choose a discrete lattice of arbitrarily large density which belongs to these hyperplanes. For instance, $\sigma_{0}\left(z_{1}, z_{2}\right)=\sigma\left(z_{1}\right) \sigma\left(z_{2}\right)$ is constructed to vanish on $\mathbb{Z}[i]^{2}$, but its zero set is the union of the complex lines $\{k\} \times \mathbb{C}$ and $\mathbb{C} \times\{l\}$ for $k, l \in \mathbb{Z}[i]$. Consequently $\sigma_{0}$ vanishes on every lattice $\epsilon \mathbb{Z}[i] \times \mathbb{Z}[i]$, which has density $\epsilon^{-2}>>1$.

Surprisingly this is not the only possibility of building such examples.

Theorem 4.1. Let $q=q_{1}+i q_{2} \in \mathbb{Z}[i]$ with $|q| \geq 2, \gamma^{2}+1 /|q|^{2}=1$ and

$$
\Lambda=\left(\begin{array}{cc}
1 & \frac{1}{q} \\
0 & \gamma
\end{array}\right) \mathbb{Z}[i]^{2}
$$

Then $\Lambda$ fails to be a set of sampling for $\mathcal{F}$.

Proof. According to Proposition 2.1 it suffices to find an $F \in \mathcal{F}_{2}^{\infty}$, such that $F(\lambda)=$ 0 for all $\lambda \in \Lambda$. We apply Proposition 3.2 to the sublattice $\Gamma=A B \mathbb{Z}[i]^{2}$ of $\Lambda$ defined by the matrix

$$
B=\left(\begin{array}{cc}
1 & -\bar{q} \\
0 & |q|^{2}
\end{array}\right)
$$

with inverse $B^{-1}=\left(\begin{array}{cc}1 & 1 / q \\ 0 & 1 /|q|^{2}\end{array}\right)$. Then $A B=\operatorname{diag}\left(1, \gamma|q|^{2}\right)$ and thus $\Gamma=A B(\mathbb{Z}[i])^{2}=$ $\mathbb{Z}[i] \times \gamma|q|^{2}(\mathbb{Z}[i])$ possesses an orthogonal basis. Then $\Delta=\operatorname{det} B=|q|^{2}$, and by 
Lemma 3.1 the cosets of $\mathbb{Z}[i]^{2} / B \mathbb{Z}[i]^{2}$ are represented by the set $\mathcal{D}=\left\{\left(0, \delta_{1}+i \delta_{2}\right) \in\right.$ $\left.\mathbb{Z}[i]^{2}: 0 \leq \delta_{1}, \delta_{2}<|q|^{2}\right\}$.

Consequently the sigma-type function of $\Gamma$ is

$$
\sigma_{\Gamma}(z)=(\sigma \otimes \sigma)\left((A B)^{-1} z\right)=\sigma\left(z_{1}\right) \sigma\left(\frac{z_{2}}{\gamma|q|^{2}}\right) .
$$

Following the recipe of (27) and (28) we partition the coset representatives $\mathcal{D}$ into

$$
\mathcal{E}_{0}=\{(0, \delta) \in \mathcal{D}: q \mid \delta\}=\{(0, \delta): \delta=q(m+i n), m, n=0, \ldots,|q|-1\},
$$

and $\mathcal{E}_{1}=\emptyset$, and $\mathcal{E}_{2}=\mathcal{D} \backslash \mathcal{E}_{0}=\{(0, \delta) \in \mathcal{D}: q \not \backslash \delta\}$.

Then card $\mathcal{E}_{0}=|q|^{2}$ and card $\mathcal{E}_{2}=|q|^{4}-|q|^{2}$. In the notation of Section 3.2 $p_{2}\left((A B)^{-1} z\right)=z_{2} /\left(\gamma|q|^{2}\right)$ and $p_{2}\left(B^{-1} \nu\right)=\delta /|q|^{2}$. Then (28) yields the explicit formula

$$
\begin{aligned}
\sigma_{\Lambda}\left(z_{1}, z_{2}\right) & =\sigma\left(z_{1}\right) \prod_{\delta \in \mathcal{E}_{2}}\left(\beta_{\delta /|q|^{2}} \sigma\right)\left(\frac{z_{2}}{\gamma|q|^{2}}\right) \\
& =\sigma\left(z_{1}\right) \prod_{\delta \in \mathcal{E}_{2}} e^{\pi \bar{\delta} z_{2} /|q|^{2}} \sigma\left(\frac{z_{2}}{\gamma|q|^{2}}-\frac{\delta}{|q|^{2}}\right) e^{-\frac{\pi}{2} \frac{|\delta|^{2}}{|q|^{4}}}
\end{aligned}
$$

By Proposition $3.2, \sigma_{\Lambda}$ vanishes on $\Lambda$.

Finally we need to check the growth of $\sigma_{\Lambda}$. Since $\beta_{w_{0}}$ is an isometry on $\mathcal{F}_{1}^{\infty}$ by (26), and since there are exactly $|q|^{4}-|q|^{2}$ factors in the product over $\mathcal{E}_{2}$, we obtain

$$
\begin{aligned}
\left|\sigma_{\Lambda}\left(z_{1}, z_{2}\right)\right| & =\left|\sigma\left(z_{1}\right)\right| \prod_{\delta \in \mathcal{E}_{2}}\left|\left(\beta_{\delta /|q|^{2}} \sigma\right)\left(\frac{z_{2}}{\gamma|q|^{2}}\right)\right| \\
& \leq \exp \left(\frac{\pi}{2}\left|z_{1}\right|^{2}\right) \prod_{\delta \in \mathcal{E}_{2}} \exp \left(\frac{\pi}{2} \frac{\left|z_{2}\right|^{2}}{\gamma^{2}|q|^{4}}\right) \\
& =\exp \left(\frac{\pi}{2}\left(\left|z_{1}\right|^{2}+\frac{|q|^{4}-|q|^{2}}{\gamma^{2}|q|^{4}}\left|z_{2}\right|^{2}\right)\right) \\
& =\exp \left(\frac{\pi}{2}\left(\left|z_{1}\right|^{2}+\left|z_{2}\right|^{2}\right),\right.
\end{aligned}
$$

where in the last identity we have used $1-\frac{1}{|q|^{2}}=\gamma^{2}$. Thus $\sigma_{\Lambda} \in \mathcal{F}_{2}^{\infty}$ and by Proposition $2.1 \Lambda$ cannot be a set of sampling for $\mathcal{F}$.

Corollary 4.2. Let $\Lambda^{\prime}$ be a lattice of the form

$$
\Lambda^{\prime}=\left(\begin{array}{ll}
\alpha & 0 \\
0 & \beta
\end{array}\right)\left(\begin{array}{cc}
1 & \frac{1}{q} \\
0 & \gamma
\end{array}\right) \mathbb{Z}[i]^{2}
$$

with $\alpha, \beta \geq 1$ and $\gamma^{2}+\frac{1}{q^{2}}=1$. Then $\Lambda^{\prime}$ is not a set of sampling for $\mathcal{F}$.

Proof. Let $D=\operatorname{diag}(\alpha, \beta)$, then $\Lambda^{\prime}=D \Lambda$ with $\Lambda$ as in Theorem 4.1.

According to Theorem 4.1 there exists a non-zero function $F \in \mathcal{F}_{2}^{\infty}$, such that $F(\lambda)=0$ for all $\lambda \in \Lambda$. Set $\tilde{F}(z)=F\left(D^{-1} z\right)$. If $\lambda^{\prime} \in \Lambda^{\prime}$, i.e. $\lambda^{\prime}=D \lambda$ for some $\lambda \in \Lambda$, we have $\tilde{F}\left(\lambda^{\prime}\right)=F\left(D^{-1} D \lambda\right)=F(\lambda)=0$ and

$$
|\tilde{F}(z)|=\left|F\left(D^{-1} z\right)\right| \leq C e^{\frac{\pi}{2}\left|D^{-1} z\right|^{2}} \leq C e^{\frac{\pi}{2}|z|^{2}} .
$$


So $\tilde{F} \in \mathcal{F}_{2}^{\infty}$ and $\tilde{F}$ vanishes on $\Lambda^{\prime}$. Therefore Proposition 2.1 implies that $\Lambda^{\prime}$ fails to be a set of sampling for $\mathcal{F}$.

Corollary 4.3. Let $p, q \in \mathbb{N}, q \geq 2, \gamma^{2}+1 / q^{2}=1$ and

$$
\Lambda=\left(\begin{array}{cc}
1 & \frac{p}{q} \\
0 & \gamma
\end{array}\right) \mathbb{Z}[i]^{2}
$$

Then $\Lambda$ fails to be a set of sampling for $\mathcal{F}$.

Proof. The proof is almost the same as of Theorem 4.1. We choose the sublattice $\Gamma$ determined by the matrix

$$
B=\left(\begin{array}{cc}
1 & -p \\
0 & q
\end{array}\right)
$$

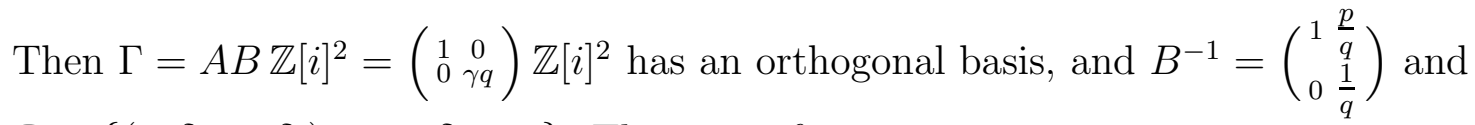
$\mathcal{D}=\left\{\left(0, \delta_{1}+i \delta_{2}\right): 0 \leq \delta_{j}<q\right\}$. The sigma function is

$$
\sigma_{\Lambda}\left(z_{1}, z_{2}\right)=\sigma\left(z_{1}\right) \prod_{\eta \in \mathcal{D} \backslash\{0\}} \beta_{\eta / q} \sigma\left(\frac{z_{2}}{\gamma q}\right)
$$

with growth

$$
\left|\sigma_{\Lambda}(z)\right| \leq e^{\frac{\pi}{2}\left|z_{1}\right|^{2}} e^{\frac{\pi}{2}\left(q^{2}-1\right) \frac{\left|z_{2}\right|^{2}}{\gamma^{2} q^{2}}}=e^{\frac{\pi}{2}|z|^{2}}
$$

because $1-q^{-2}=\gamma^{2}$.

One may wonder how we chose the sublattice $\Gamma$. Our guiding principle was to find a sublattice with an orthogonal basis because in this case the norm $\left\|(A B)^{-1}\right\|_{\text {op }}$ is minimized. We do not know how to make Theorem 4.1 work in greater generality.

Consider the one-parameter family of matrices $A_{t}=\left(\begin{array}{cc}1 & \frac{2}{5} \\ 0 & t\end{array}\right)$ with $t^{2}+(2 / 5)^{2} \geq 1$ (as we assume a reduced basis). Corollary 4.3 and 4.2 say that the lattices $\Lambda_{t}=$ $A_{t} \mathbb{Z}[i]^{2}$ fail to be sampling for $t \geq \sqrt{24} / 5$, but we do not know what happens for $\sqrt{21} / 5 \leq t<\sqrt{24} / 5$.

\section{A Weak Sampling Formula}

Theorem 4.1 and Corollaries 4.2 and 4.3 show that many lattices with density $>1$ fail to be sampling sets for $\mathcal{F}_{2}^{2}$. By contrast, we have not succeeded to use the interpolating functions of Proposition 3.3 to prove positive results about sampling lattices. Nevertheless, we can prove a slightly weaker sampling theorem which holds even for dimensions $d>2$.

This section is a symbiosis of complex analysis (construction of interpolating functions) and Gabor analysis (duality theory of Gabor frames). In fact, we translate a weak reconstruction formula of Feichtinger and Zimmermann [7] into a Lagrange-type reconstruction for entire functions.

For the discussion we need some more background from Gabor analysis. The modulation space $M^{1}\left(\mathbb{R}^{d}\right)$ is the subspace of $L^{2}\left(\mathbb{R}^{d}\right)$ for which the norm

$$
\|h\|_{M^{1}}=\int_{\mathbb{R}^{2 d}}\left|\left\langle h, \pi_{z} \phi\right\rangle\right| d z
$$


is finite, where $\phi$ is the normalized Gaussian. Its dual is the space of tempered distributions such that $\sup _{z \in \mathbb{R}^{2 d}}\left|\left\langle h, \pi_{z} \phi\right\rangle\right|<\infty$. Then $\left(M^{1}\right)^{*}=M^{\infty}$, and the duality is given via the Bargmann transform as

$$
\langle h, k\rangle_{M^{1} \times M^{\infty}}=\int_{\mathbb{C}^{d}} B h(z) \overline{B k(z)} e^{-\pi|z|^{2}} d z=\langle B h, B k\rangle .
$$

Introducing the Fock space $\mathcal{F}_{d}^{1}$ consisting of all entire functions with finite norm

$$
\|F\|_{\mathcal{F}_{d}^{1}}=\int_{\mathbb{C}^{d}}|F(z)| e^{-\pi|z|^{2} / 2} d z<\infty
$$

we can identify $M^{1}$ and $M^{\infty}$ as the pre-images of $\mathcal{F}_{d}^{1}$ and $\mathcal{F}_{d}^{\infty}$ of the Bargmann transform [10].

The detailed analysis of the duality theory of Gabor frames led Feichtinger and Zimmermann [7] to the theory of weak dual pairs. We will apply the following version of the duality theory [7, Thm. 3.5.12].

Proposition 5.1 (Weak duality). Let $\Lambda \subseteq \mathbb{R}^{2 d}$ be a lattice with adjoint lattice $\Lambda^{\circ}$, $g \in M^{1}\left(\mathbb{R}^{d}\right)$ and $\gamma \in M^{\infty}\left(\mathbb{R}^{d}\right)$. Then the following are equivalent:

(i) Biorthogonality on the adjoint lattice:

$$
\frac{1}{\operatorname{vol}(\Lambda)}\left\langle\gamma, \pi_{\mu} g\right\rangle=\delta_{\mu, 0} \text { for all } \mu \in \Lambda^{\circ} .
$$

(ii) For every $f, h \in M^{1}\left(\mathbb{R}^{d}\right)$ we have

$$
\langle f, h\rangle=\sum_{\lambda \in \Lambda}\left\langle f, \pi_{\lambda} g\right\rangle\left\langle\pi_{\lambda} \gamma, h\right\rangle
$$

with absolute convergence of the sum.

The identity (32) can be interpreted as a reconstruction formula

$$
f=\sum_{\lambda \in \Lambda}\left\langle f, \pi_{\lambda} g\right\rangle \pi_{\lambda} \gamma
$$

for $f \in M^{1}$, but with convergence in the weak*-topology on $M^{\infty}$.

By applying the Bargmann transform, Proposition 5.1 with $g(x)=2^{d / 4} e^{-\pi|x|^{2}}$ is translated into the following Lagrange interpolation formula for $\mathcal{F}_{d}^{1}$.

Corollary 5.2. Let $\Lambda \subseteq \mathbb{C}^{d}$ be a complex lattice with adjoint lattice $\Lambda^{\circ} \subseteq \mathbb{C}^{d}$ and $\tau \in \mathcal{F}_{d}^{\infty}$. Then the following are equivalent:

(i) $\tau$ is interpolating on $\Lambda^{\circ}$.

(ii) For all $F \in \mathcal{F}_{d}^{1}$

$$
F(z)=\sum_{\lambda \in \Lambda} F(\lambda) e^{\pi \bar{\lambda} \cdot z} \tau(z-\lambda) e^{-\pi|\lambda|^{2}}
$$

The series expansion converges weakly in the sense that for all $H \in \mathcal{F}_{d}^{1}$

$$
\int_{\mathbb{C}^{d}} F(z) \overline{H(z)} e^{-\pi|z|^{2}} d z=\sum_{\lambda \in \Lambda} F(\lambda) e^{-\pi|\lambda|^{2}} \int_{\mathbb{C}^{d}} e^{\pi \bar{\lambda} \cdot z} \tau(z-\lambda) \overline{H(z)} e^{-\pi|z|^{2}} d z .
$$


Proof. This follows from the properties of the Bargmann transform: $\langle f, h\rangle=$ $\int_{\mathbb{C}^{d}} B f(z) \overline{B h(z)} e^{-\pi|z|^{2}} d z$, and $\left\langle f, \pi_{\lambda} \phi\right\rangle=B f(\lambda) e^{-\pi|\lambda|^{2} / 2}$ and the fact that the Bargmann transform is an isomorphism from $M^{1}$ onto $\mathcal{F}_{d}^{1}$ and from $M^{\infty}$ onto $\mathcal{F}_{d}^{\infty}$.

Combining Corollary 5.2 with the construction of interpolating functions we obtain the following Lagrange interpolation formula.

Theorem 5.3. Let $\Lambda=S \mathbb{Z}[i]^{d}$ where $S$ is an upper triangular matrix with diagonal $\left(\gamma_{1}, \ldots, \gamma_{d}\right)$ as in (16). Assume that $\max _{j=1, \ldots, d} \gamma_{j}=1$.

(i) Then there exists $\gamma \in M^{\infty}$ such that $\frac{1}{\operatorname{vol}(\Lambda)}\left\langle\gamma, \pi_{\mu} g\right\rangle=\delta_{\mu, 0}$ for all $\mu \in \Lambda^{\circ}$ and the weak reconstruction formula (32) holds.

(ii) Equivalently, there exists an interpolating function $\tau \in \mathcal{F}_{d}^{\infty}$ for the adjoint lattice $\Lambda^{\circ}$, such that the weak Lagrange interpolation formula (33) holds. In particular, $\Lambda$ is a set of uniqueness for $\mathcal{F}_{d}^{1}$.

Proof. The adjoint lattice of $\Lambda$ is $\left(S^{-1}\right)^{*} \mathbb{Z}[i]^{d}$, and the diagonal of $\left(S^{-1}\right)^{*}$ is $\left(\gamma_{1}^{-1}, \ldots, \gamma_{d}^{-1}\right)$. Then the entire function

$$
\tau(z)=\tau\left(z_{1}, z_{2}, \ldots, z_{d}\right)=\prod_{j=1}^{d} \frac{\sigma\left(\gamma_{j} z_{j}\right)}{z_{j}}
$$

is interpolating for $\Lambda^{\circ}$. This was already proved in [9]. Furthermore, by the growth estimate for the Weierstrass sigma-function (11) we have $\left|\frac{\sigma\left(\gamma_{j} z_{j}\right)}{z_{j}}\right| \leq C e^{\pi \gamma_{j}^{2}\left|z_{j}\right|^{2} / 2}$. Consequently,

$$
|\tau(z)| \leq C^{\prime} e^{\pi\left(\max \gamma_{j}^{2}\right)|z|^{2} / 2}
$$

Consequently, $\tau \in \mathcal{F}_{d}^{\infty}$. However, since $\gamma_{l}=1$ for some $l$, we have $\tau \notin \mathcal{F}_{d}^{2}$. We now choose $\gamma \in M^{\infty}$, so that $B \gamma=\tau$. The statement now follows from the assertion of Proposition 5.1 and Corollary 5.2 .

Obviously our results are far from complete and should be considered a collection of expected and of surprising examples. At this time we do not even understand

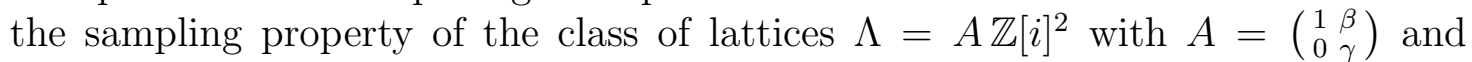
$|\beta|^{2}+\gamma^{2}=1$. As we have seen, for certain values of $\beta, \beta=1 / q, q \in \mathbb{Z}[i],|q| \geq 2$, the lattice $\Lambda$ is not sampling, but nothing else is known.

\section{REFERENCES}

[1] Akhiezer, N. I. Elements of the theory of elliptic functions. Translated from the second Russian edition by H. H. McFaden. Translations of Mathematical Monographs, 79. American Mathematical Society, Providence, RI, 1990. viii+237 pp.

[2] V. Bargmann. On a Hilbert space of analytic functions and an associated integral transform. Comm. Pure Appl. Math., 14:187-214, 1961.

[3] V. Bargmann, P. Butera, L. Girardello, and J. R. Klauder. On the completeness of coherent states. Rep. Math. Phys., 2:221-228, 1971.

[4] M. Faulhuber and S. Steinerberger. Optimal Gabor frame bounds for separable lattices and estimates for Jacobi theta functions. J. Math. Anal. Appl., 445(1):407-422, 2017.

[5] H. G. Feichtinger and K. Gröchenig. Banach spaces related to integrable group representations and their atomic decompositions. I. J. Functional Anal., 86(2):307-340, 1989. 
[6] H. G. Feichtinger and W. Kozek. Quantization of TF lattice-invariant operators on elementary LCA groups. In Gabor analysis and algorithms, pages 233-266. Birkhäuser Boston, Boston, MA, 1998.

[7] H. G. Feichtinger and G. Zimmermann. A Banach space of test functions for Gabor analysis. In Gabor analysis and algorithms, pages 123-170. Birkhäuser Boston, Boston, MA, 1998.

[8] G. B. Folland. Harmonic Analysis in Phase Space. Princeton Univ. Press, Princeton, NJ, 1989.

[9] Gröchenig, K. Multivariate Gabor frames and sampling of entire functions of several variables. Appl. Comput. Harmon. Anal. 31(2):218-227, 2011.

[10] K. Gröchenig. Foundations of time-frequency analysis. Birkhäuser Boston Inc., Boston, MA, 2001.

[11] K. Gröchenig. Gabor frames without inequalities. Int. Math. Res. Not. IMRN 2007, no. 23, Art. ID rnm111, 21 pp.

[12] K. Gröchenig. The mystery of Gabor frames. J. Fourier Anal. Appl. 20(4):865- 895, 2014.

[13] K. Ireland and M. Rosen. A classical introduction to modern number theory, volume 84 of Graduate Texts in Mathematics. Springer-Verlag, New York, 1990.

[14] A. J. E. M. Janssen. Duality and biorthogonality for Weyl-Heisenberg frames. J. Fourier Anal. Appl., 1(4):403-436, 1995.

[15] P. Lelong and L. Gruman. Entire functions of several complex variables, volume 282 of Grundlehren der Mathematischen Wissenschaften. Springer-Verlag, Berlin, 1986.

[16] Hayman, W. The local growth of the power series: A survey of the Wiman-Valiron method. Canad. Math. Bull. 17:317-358, 1974.

[17] Heil, C. History and evolution of the density theorem for Gabor frames. J. Fourier Anal. Appl. 13(2): 113-166, 2007.

[18] A. K. Lenstra, H. W. Lenstra, Jr., and L. Lovász. Factoring polynomials with rational coefficients. Math. Ann., 261(4):515-534, 1982.

[19] Y. I. Lyubarskii. Frames in the Bargmann space of entire functions. In Entire and subharmonic functions, pages 167-180. Amer. Math. Soc., Providence, RI, 1992.

[20] Y. I. Lyubarskii and A. Rashkovskii. Complete interpolating sequences for Fourier transforms supported by convex symmetric polygons. Ark. Mat., 38(1):139-170, 2000.

[21] X. Massaneda and P. J. Thomas. Interpolating sequences for Bargmann-Fock spaces in $\mathbb{C}^{n}$. Indag. Math. (N.S.), 11(1):115-127, 2000.

[22] J. Ortega-Cerdá and K. Seip. Beurling-type density theorems for weighted $L^{p}$ spaces of entire functions. J. Anal. Math. 75 (1998), 247266.

[23] D. E. Papush. The growth of entire functions with "plane" zeros. Teor. Funktsiu Funktsional. Anal. i Prilozhen., (48):117-125, 1987.

[24] D. E. Papush. Interpolation with discrete sets in $\mathbf{C}^{l}$. J. Soviet Math., 59(1):666-674, 1992. Translation of Teor. Funktsiú Funktsional. Anal. i Prilozhen. No. 55 (1991), 113-124.

[25] A. M. Perelomov. Remark on the completeness of the coherent state system. Teoret. Mat. Fiz., 6(2):213-224, 1971.

[26] G. E. Pfander and P. Rashkov. Remarks on multivariate Gaussian Gabor frames. Monatsh. Math., 172(2):179-187, 2013.

[27] A. Ron and Z. Shen. Weyl-Heisenberg frames and Riesz bases in $L_{2}\left(\mathbb{R}^{d}\right)$. Duke Math. J., 89(2):237-282, 1997.

[28] L. I. Ronkin. Introduction to the theory of entire functions of several variables. American Mathematical Society, Providence, R.I., 1974. Translated from the Russian by Israel Program for Scientific Translations, Translations of Mathematical Monographs, Vol. 44.

[29] K. Seip. Density theorems for sampling and interpolation in the Bargmann-Fock space. I. $J$. Reine Angew. Math., 429:91-106, 1992.

[30] K. Seip and R. Wallstén. Density theorems for sampling and interpolation in the BargmannFock space. II. J. Reine Angew. Math., 429:107-113, 1992. 
[31] B. Simon. Basic complex analysis. A Comprehensive Course in Analysis, Part 2A. American Mathematical Society, Providence, RI, 2015.

[32] T. Strohmer and S. Beaver. Optimal ofdm design for time-frequency dispersive channels. Communications, IEEE Transactions on, 51(7):1111-1122, July 2003.

Faculty of Mathematics, University of Vienna, Oskar-Morgenstern-Platz 1, A-1090 Vienna, Austria

E-mail address: karlheinz.groechenig@univie.ac.at

Department of Mathematical Sciences, Norwegian University of Science and Technology, NO-7491, Trondheim, Norway

E-mail address: yura@math.ntnu.no 